\title{
Kinderneuroradiologisches Netzwerk
}

Interdisziplinäre Falldiskussion für Lehrende und Lernende

Die pädiatrische Neuroradiologie bildet einen wichtigen und komplexen Teil der pädiatrischen Radiologie und der Neuroradiologie. Erfahrungsgemäß gibt es in jedem Zentrum meist 1-2 (Neuro-/pädiatrische) Radiologinnen und Radiologen, die sich schwerpunktmäßig mit diesem Gebiet befassen, sodass der kollegiale Austausch begrenzt ist und in kleineren Zentren möglicherweise auch gar nicht möglich ist.

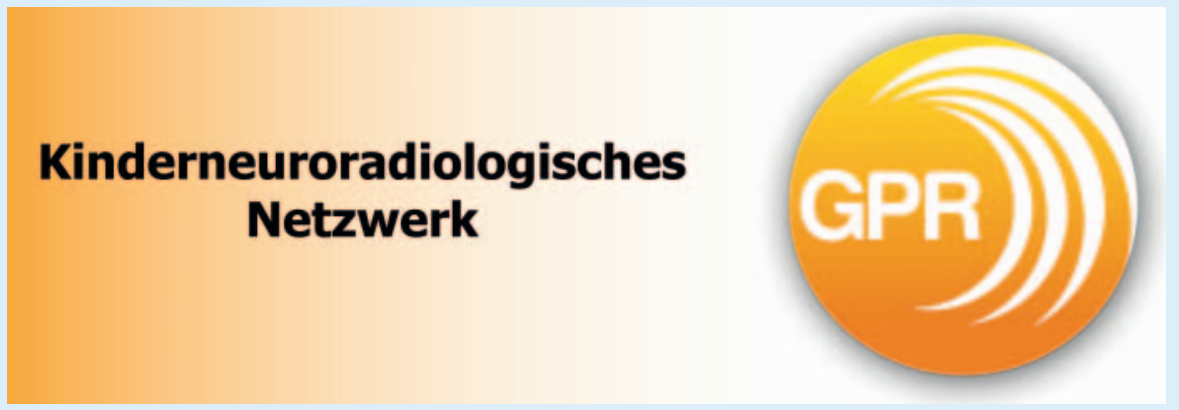

Da jeden von uns jedoch immer wieder interessante und herausfordernde Fälle erreichen, ist ein kollegialer Austausch nicht nur zur Falldiskussion im Rahmen der Diagnose- findung von Interesse, sondern auch zur Fort- und Weiterbildung anzustreben. 
Das Kinderneuroradiologische Netzwerk richtet sich demnach explizit an Interessierte der verschiedensten Fachrichtungen:

- Kinderradiologie

- Neuroradiologie

- Radiologie

- Pädiatrie

- Genetik

- (Kinder-)Chirurgie

- Pränatal- und Geburtsmedizin

- (pädiatrische) Intensivmedizin

- u.v.m.

Ausdrücklich ansprechen möchten wir junge Kolleginnen und Kollegen und sie ermuntern, schwierige oder unklare Fälle mit uns zu teilen. Durch die gemeinsame Diskussion im Rahmen des Netzwerks kann ein effektiver Austausch von Erfahrungen und Kenntnissen geschehen, der jungen, aber auch erfahrenen
Kolleginnen und Kollegen einen großen Benefit für die eigene Arbeit geben kann.

Melden Sie sich bei uns! Schildern Sie uns Ihre Fälle, die Sie gerne mit Kolleginnen und Kollegen besprechen möchten.

\section{Kontakt:}

Dr. Gabriele Hahn: gabriele.hahn@uniklini kum-dresden.de

Dr. Monika Huhndorf: Monika.Huhndorf@ uksh.de

GPR-Geschäftsstelle: buero@kinder-radiolo gie.org (bei organisatorischen Fragen)

\section{Die Termine 2022:}

Donnerstag, 27. Januar 2022 von 16:00 Uhr bis 17:30 Uhr
Donnerstag, 28., April 2022 von 16:00 Uhr bis 17:30 Uhr

Donnerstag, 30. Juni 2022 von 16:00 Uhr bis 17:30 Uhr

Donnerstag, 27. Oktober 2022 von 16:00 Uhr bis 17:30 Uhr

Die Teilnahme ist kostenfrei, eine Anmeldung über Zoom aber jeweils erforderlich.

Den Registrierungslink sowie das aktuelle Programm finden Sie ab Dezember 2021 auf www.kinder-radiologie.org.

Für Rückfragen steht Ihnen die Geschäftsstelle gerne zur Verfügung. 